\title{
SOME CONDITIONS ON AN OPERATOR IMPLYING NORMALITY. II
}

\author{
S. K. BERBERIAN
}

ABstract. Conditions are given under which an operator with countable spectrum is normal, and applications are made to polynomially compact operators.

This paper is a continuation of [1], using techniques derived in [2] and [3]. We refer to either [2] or [3] for notations and terminology, but for convenience we repeat some of the definitions.

An operator $T$ is said to satisfy condition $\left(G_{1}\right)$ if $(T-\lambda I)^{-1}$ is normaloid (equivalently, $\left\|(T-\lambda I)^{-1}\right\|=[\operatorname{dist}(\lambda, \sigma(T))]^{-1}$ ) for all $\lambda \notin \sigma(T)$; it follows that such an operator is isoloid, i.e., every isolated point of $\sigma(T)$ is an eigenvalue of $T$. We also consider the following conditions on an operator $T$ :

$\left(\alpha^{\prime}\right) T$ is reduction- $\left(G_{1}\right)$, i.e., every direct summand of $T$ satisfies $\left(G_{1}\right)$.

$\left(\alpha^{\prime \prime}\right) T$ is restriction-convexoid, i.e., the restriction of $T$ to any invariant subspace is convexoid.

$\left(\alpha^{\prime \prime \prime}\right) T$ is reduction-isoloid, i.e., every direct summand of $T$ is isoloid.

It is known [2] that seminormal $\Rightarrow\left(\alpha^{\prime}\right) \Rightarrow\left(\alpha^{\prime \prime \prime}\right)$ and that hyponor$\mathrm{mal} \Rightarrow\left(\alpha^{\prime \prime}\right) \Rightarrow\left(\alpha^{\prime \prime \prime}\right)$. One says that Weyl's theorem holds for an operator $T$ if $\omega(T)=\sigma(T)-\pi_{00}(T)$, where $\omega(T)$ is the Weyl spectrum of $T$ and $\pi_{00}(T)$ is the set of isolated points of $\sigma(T)$ that are eigenvalues of finite multiplicity.

A normal operator $T$ is said (for the obvious matricial reason) to be diagonal if the eigenvectors of $T$ are total, i.e., if the underlying Hilbert space is the closed linear span (=orthogonal direct sum) of the eigenspaces of $T[\mathbf{5}$, p. 29]. \{The term " $T$ has pure point spectrum" is fairly standard for this, but it is an unreliable heuristic guide because the property of being diagonal neither implies nor is implied by the condition $\sigma(T)=\pi_{0}(T)$. $\}$ It is a folk theorem that every normal operator with countable spectrum is diagonal (a stronger result is given in Theorem 1).

Theorem 3 of $[1]$ is as follows: If $T$ satisfies $\left(\alpha^{\prime \prime}\right)$ and $\sigma(T)$ has at most finitely many accumulation points (i.e., the derived set $\sigma(T)^{\prime}$ is

Received by the editors January 12, 1970 and, in revised form, March 10, 1970. AMS 1969 subject classifications. Primary 4740, 4730.

Key words and phrases. Hilbert space, normal operator, Weyl spectrum. 
finite), then $T$ is a diagonal normal operator. It is clear from the preliminaries to this result that if $T$ satisfies $\left(\alpha^{\prime}\right)$ and $\sigma(T)$ has at most one accumulation point, then $T$ is a diagonal normal operator [1, proofs of Lemmas 3 and 4]. This suggests the question: If $T$ satisfies $\left(\alpha^{\prime}\right)$ and $\sigma(T)$ has at most finitely many accumulation points, does it follow that $T$ is a diagonal normal operator? The answer is strongly affirmative:

Theorem 1. If $T$ satisfies $\left(\alpha^{\prime}\right)$ and $\sigma(T)$ is countable, then $T$ is a diagonal normal operator.

Informally, the interest of this result is that condition $\left(\alpha^{\prime}\right)$ requires only knowledge of the reducing subspaces, whereas $\left(\alpha^{\prime \prime}\right)$ requires knowledge of all the invariant subspaces. Of course $\sigma(T)$ is countable whenever $\sigma(T)^{\prime}$ is finite; better yet, $\sigma(T)$ is countable if and only if $\sigma(T)^{\prime}$ is countable (because the set of isolated points of $\sigma(T)$ is countable $[6$, p. 147, II $])$.

Proof of Theorem 1. Let $\delta$ be the set of all eigenvalues $\lambda$ of $T$ such that $N(T-\lambda I)$ reduces $T$, that is,

$$
\delta=\left\{\lambda \in \pi_{0}(T): N(T-\lambda I) \subset N\left(T^{*}-\lambda^{*} I\right)\right\} .
$$

If $\mathscr{T C}$ is the closed linear span of the subspaces $N(T-\lambda I)(\lambda \in \delta)$, then $\mathfrak{T}$ reduces $T$, and $T_{1}=T \mid \mathfrak{T}$ is normal and diagonal. If $\mathfrak{T}^{\perp}=\{0\}$ we are through. Assuming to the contrary that $\mathfrak{T}^{\perp} \neq\{0\}$, let $T_{2}=T \mid \mathfrak{T C}^{\perp}$. Then $T=T_{1} \oplus T_{2}, \pi_{0}\left(T_{1}\right)=\delta$, and $\pi_{0}\left(T_{2}\right)=\pi_{0}(T)-\delta$ [3, Proposition 4.1]. Since $\sigma(T)=\sigma\left(T_{1}\right) \cup \sigma\left(T_{2}\right), \sigma\left(T_{2}\right)$ is also countable, therefore $\sigma\left(T_{2}\right)$ has at least one isolated point $\lambda$ (otherwise $\sigma\left(T_{2}\right)$ would be dense-in-itself, hence perfect, hence uncountable $[6$, p. 156, VI]). Since $T_{2}$ satisfies $\left(G_{1}\right), \lambda$ is an eigenvalue of $T_{2} ;$ moreover, $N\left(T_{2}-\lambda I\right)$ $=N\left(T_{2}^{*}-\lambda^{*} I\right) \quad\left[2\right.$, Example 6]. But $N(T-\lambda I)=N\left(T_{2}-\lambda I\right) \quad$ [3, Proposition 4.1] and $N\left(T_{2}^{*}-\lambda^{*} I\right) \subset N\left(T^{*}-\lambda^{*} I\right)$ (because $\left(T \mid \mathscr{T}^{\perp}\right)^{*}$ $\left.=T^{*} \mid \mathfrak{T}^{\perp}\right)$, therefore $N(T-\lambda I) \subset N\left(T^{*}-\lambda^{*} I\right)$. This shows that $\lambda \in \delta$, whereas $\lambda \in \pi_{0}\left(T_{2}\right)=\pi_{0}(T)-\delta$, a contradiction.

Since the set of isolated points of $\sigma(T)$ is countable, so is $\pi_{00}(T)$. If, moreover, Weyl's theorem holds for $T$, then $\sigma(T)=\omega(T) \cup_{\pi_{00}}(T)$ shows that $\sigma(T)$ is countable if and only if $\omega(T)$ is countable. Citing Theorem 1, we have:

Corollary. If (i) T satisfies ( $\left.\alpha^{\prime}\right)$, (ii) Weyl's theorem holds for $T$, and (iii) $\omega(T)$ (equivalently, $\sigma(T)$ ) is countable, then $T$ is a diagonal normal operator.

A special case [4, Proposition 4]: If $T$ is seminormal and $\sigma(T)$ is countable, then $T$ is normal (hence diagonal). \{More generally, $\mathrm{C}$. R. 
Putnam has recently shown that a seminormal operator whose spectrum has planar measure 0 is normal [7].\} In turn, this result can be improved in another direction:

TheOREM 2. If (i) $T$ satisfies ( $\left.\alpha^{\prime \prime \prime}\right)$, (ii) $T$ is reduced by each of its eigenspaces, and (iii) $\omega(T)$ (equivalently, $\sigma(T)$ ) is countable, then $T$ is a diagonal normal operator.

Proof. Since (i) and (ii) imply that Weyl's theorem holds for $T$ [2], $\sigma(T)$ is countable if and only if $\omega(T)$ is countable.

Let $\delta=\pi_{0}(T)$ and let $\Re$ be the closed linear span of the subspaces $N(T-\lambda I)(\lambda \in \delta)$; it is to be shown that $\mathfrak{N}^{\perp}=\{0\}$. Assuming to the contrary, write $T=T_{1} \oplus T_{2}$ as in [3, Proposition 4.1]; in particular, $\sigma\left(T_{2}\right)$ is nonempty and countable, hence it has at least one isolated point $\lambda$. Citing $\left(\alpha^{\prime \prime \prime}\right)$, we have the absurdity $\lambda \in \pi_{0}\left(T_{2}\right)=\pi_{0}(T)-\delta$ $=\varnothing$.

Left unanswered is the following tantalizing question: If $T$ satisfies $\left(\alpha^{\prime \prime}\right)$ and $\sigma(T)$ is countable, is $T$ normal? It would suffice (by an obvious exhaustion argument) to show that $T$ has a reducing eigenspace.

We note three applications to polynomially compact operators; these extend results in $[3$, Theorems $6.5,6.7]$, the new feature being that it is not hypothesized that $T$ is reduced by any of its eigenspaces:

Corollary 1. If (i) T satisfies either $\left(\alpha^{\prime}\right)$ or $\left(\alpha^{\prime \prime}\right)$, (ii) Weyl's theorem holds for $T$, and (iii) $\omega(T)$ is finite, then $T$ is normal and polynomially compact.

Proof. Since $\omega(T)=\sigma(T)-\pi_{00}(T) \supset \sigma(T)^{\prime}, \sigma(T)^{\prime}$ is finite. If $T$ satisfies $\left(\alpha^{\prime}\right)$ then $T$ is normal by the corollary of Theorem 1 . If $T$ satisfies $\left(\alpha^{\prime \prime}\right)$ then $T$ is normal by [1, Theorem 3]. In either case, the finiteness of $\omega(T)$ implies that $T$ is polynomially compact $[3$, Theorem $6.4]$.

Corollary 2. If (i) $T$ satisfies ( $\left.\alpha^{\prime}\right)$ and (ii) $T$ is polynomially compact, then $T$ is normal and $\omega(T)$ is finite.

Proof. Let $p$ be a nonzero polynomial such that $p(T)$ is compact. If the underlying Hilbert space is finite-dimensional, then $T$ is normal by $[1$, Theorem 2]. Otherwise, $p$ is nonconstant; since $p(\sigma(T))$ $=\sigma(p(T))$ is countable, so is $\sigma(T)$, therefore $T$ is normal by Theorem 1. Finally, $\omega(T)$ is finite by $[3$, Theorem 6.4].

Combining Corollaries 1 and 2:

Corollary 3. Suppose (i) T satisfies $\left(\alpha^{\prime}\right)$, and (ii) Weyl's theorem 
holds for $T$. Then $T$ is polynomially compact if and only if $\omega(T)$ is finite, and in this case $T$ is normal.

We conclude with a new, highly combinatorial proof of Gilfeather's structure theorem for polynomially compact normal operators [4]:

THEOREM 3. If $T$ is a normal operator, the following conditions are equivalent: (a) $T$ is polynomially compact; (b) $\omega(T)$ is finite; (c) $T$ is the orthogonal direct sum of finitely many thin normal operators, i.e.,

$$
T=\left(K_{1}+\lambda_{1} I\right) \oplus \cdots \oplus\left(K_{n}+\lambda_{n} I\right),
$$

where the $K_{i}$ are compact normal operators.

Proof. (c) $\Rightarrow(\mathrm{b})$ : If $T$ has the indicated structure, then by normality [2, Example 5] $\omega(T)=\bigcup_{1}^{n} \omega\left(K_{i}+\lambda_{i} I\right) \subset\left\{\lambda_{1}, \cdots, \lambda_{n}\right\}$.

(a) $\Leftrightarrow$ (b): See [3, Theorem 6.4]

(b) $\Rightarrow(\mathrm{c})$ : The proof is by induction on card $\omega(T)$. If $\omega(T)=\{\lambda\}$ then $\omega(T-\lambda I)=\{0\}$, and it follows from normality that $T-\lambda I$ is compact [3, remarks following Corollary 6.3]. Suppose card $\omega(T)=n>1$ and assume the theorem true for cardinality $<n$. Since $T$ is diagonal (e.g., by Theorem 1), $\sigma(T)=\mathrm{Cl} \pi_{0}(T)$ [5, Problem 48], thus every point of $\sigma(T)$ is either an eigenvalue or an accumulation point of eigenvalues (or both).

Choose $\lambda_{1}, \lambda_{2} \in \omega(T), \lambda_{1} \neq \lambda_{2}$; let $U$ be a closed neighborhood of $\lambda_{1}$ that excludes $\lambda_{2}$, and define

$$
\delta_{1}=U \cap \pi_{0}(T), \quad \delta_{2}=\pi_{0}(T)-\delta_{1} .
$$

Obviously $\lambda_{2} \notin \mathrm{Cl} \delta_{1}$. Also $\lambda_{1} \notin \delta_{2}$ (this is trivial if $\lambda_{1}$ is not an eigenvalue, whereas if $\lambda_{1}$ is an eigenvalue then $\left.\lambda_{1} \in \delta_{1}\right)$, and clearly $\lambda_{1} \notin \delta_{2}{ }^{\prime}$, therefore $\lambda_{1} \notin \mathrm{Cl} \delta_{2}$.

For $k=1,2$, let $\mathfrak{T}_{k}$ be the closed linear span of the eigenspaces $N(T-\lambda I)\left(\lambda \in \delta_{k}\right)$; since $T$ is diagonal and $\pi_{0}(T)$ is the disjoint union of $\delta_{1}$ and $\delta_{2}$, the underlying Hilbert space is the orthogonal direct sum of $\mathfrak{T}_{1}$ and $\mathfrak{T}_{2}$. Thus, writing $T_{k}=T \mid \Re_{k}$, we have $T=T_{1} \oplus T_{2}$. Since $\lambda_{2} \notin \mathrm{Cl} \delta_{1}=\sigma\left(T_{1}\right)$ and $\lambda_{1} \notin \mathrm{Cl} \delta_{2}=\sigma\left(T_{2}\right)$, all the more $\lambda_{2} \notin \omega\left(T_{1}\right)$ and $\lambda_{1} \notin \omega\left(T_{2}\right)$; it then follows from $\omega(T)=\omega\left(T_{1}\right) \cup \omega\left(T_{2}\right)$ that the induction hypothesis is applicable to both $T_{1}$ and $T_{2}$.

\section{REFERENCES}

1. S. K. Berberian, Some conditions on an operator implying normality, Math. Ann. 184 (1970), 188-192.

2. - An extension of Weyl's theorem to a class of not necessarily normal operators, Michigan Math. J. 16 (1969), 273-279.

3. - The Weyl spectrum of an operator, J. Math. Mech. (to appear). 
4. F. Gilfeather, The structure and asymptotic behavior of polynomially compact operators, Proc. Amer. Math. Soc. 25 (1970), 127-134.

5. P. R. Halmos, A Hilbert space problem book, Van Nostrand, Princeton, N. J., 1967. MR $34 \# 8178$.

6. F. Hausdorff, Set theory, Reprint, Dover, New York, 1944; English transl., Chelsea, New York, 1957. MR 7, 419; MR 19, 111.

7. C. R. Putnam, An inequality for the area of hyponormal spectra, (to appear).

University of Texas, Austin, Texas 78712 